\title{
Le complément de la localisation spatiale : entre argument et adjoint
}

\author{
Anne Carlier \\ CNRS UMR 8094 Lattice (ENS, Paris) \\ Université de Lille / Nord de France, Valenciennes \\ Anne.Carlier@univ-valenciennes.fr \\ Laure Sarda \\ CNRS UMR 8094 Lattice (ENS, Paris) \\ Laure.Sarda@ens.fr
}

\section{La distinction entre arguments et adjoints}

Quel que soit le cadre théorique que l'on adopte, il est coutume de séparer les constituants qui entretiennent un rapport morphosyntaxique étroit avec le verbe et les constituants plus périphériques par rapport au verbe. Pour caractériser les premiers, on utilise le terme d'arguments du verbe, d'actants ou d'éléments de valence. On départage les arguments ou actants par rapport aux adjoints ou circonstants sur la base d'un certain nombre de critères dont les contraintes de présence, de forme et de position des constituants concernés ainsi que la présence de morphèmes verbaux tels les marques d'accord et les pronoms clitiques. L'application de ces critères met en évidence une zone grise pour laquelle les critères donnent des résultats moins univoques (Dowty 2003). Lazard (1996) donne une place à cette zone grise dans sa classification en séparant les contraintes de présence et les contraintes de forme. Outre l'actant obligatoire exigé par tout verbe quel qu'il soit - c'est le cas du sujet dont la présence est exigée par tout verbe conjugué en français - il distingue trois types d'actants.

- Les actants requis et régis : leur présence est exigée et leur forme est contrainte par le verbe (rencontrer un ami, une difficulté).

- Les actants régis : leur présence n'est pas exigée, mais leur forme est contrainte par le verbe (je pense, je pense à mon ami).

- Les actants requis : leur forme est libre, mais leur présence est exigée par le verbe (Pierre habite la campagne / Pierre habite à la campagne / Pierre habite dans un château). Ces derniers occupent une position plus périphérique par rapport au verbe que les actants régis : alors qu'au maximum un seul constituant peut occuper la position syntaxique correspondant à un actant régi, il est possible d'avoir plusieurs constituants dans le cas d'un actant requis (Pierre habite à la campagne dans un château chez sa grand-mère).

Les constituants nominaux ni régis ni requis sont des circonstants.

La notion d'actant requis nécessite une précision : un élément absent n'est pas nécessairement inexistant, mais peut être latent. Par rapport aux éléments absents, Fillmore \& Kay (1996) distinguent trois cas :

- l'instanciation nulle libre, impliquant un référent non précisé, pouvant éventuellement être reconstitué à partir du contexte ou de la situation : Marie a affiché le message (sur le panneau);

- l'instanciation nulle indéfinie, impliquant un référent indéfini : Pierre mange ;

- l'instanciation nulle définie, impliquant un référent identifiable : Je n'ai pas terminé (ce travail / ce livre / mon repas).

Dans le cas de l'instanciation nulle définie, nous avons à faire à un actant requis. Se pose la question de savoir si les autres cas doivent être considérés comme des actants.

Il a été avancé entre autres par Goldberg (1995, 2006 : 39) que la structure argumentale doit être associée non pas au verbe en tant qu'unité du lexique, mais à la construction dans laquelle entre le verbe. Cette 
approche constructionnelle présente l'avantage de ne pas considérer la multiplicité de structures argumentales pour un même verbe comme une anomalie, mais de formuler au contraire des règles associant la forme des différentes constructions à une interprétation.

(1) a. Pierre coupe le pain.

b. Pierre coupe le pain en morceaux / en quatre.

c. Pierre lui/ $\varnothing$ coupe une tranche de pain.

d. Pierre lui $/ * \varnothing$ coupe la parole.

e. Pierre se coupe le doigt.

f. Pierre se coupe au doigt.

g. Quand l'herbe est mouillée, elle se coupe plus facilement. ((tour pronominal médio-pass.)

(2) a. Le traîneau glissait facilement sur la neige dure. (Hémon) (intransitif)
b. Maman glissa une pièce à chacun. (Rouaud)
(transitif direct et indirect en $\grave{a} /$ au datif)

Ainsi, selon Goldberg (2006: 20), le rapport entre construction et interprétation peut être représenté pour $(1 \mathrm{c}-\mathrm{d})$ et $(2 \mathrm{~b})$ par le schéma suivant, où la ligne plaine signifie que la position argumentale dans la construction est associée à un rôle thématique dont dispose le verbe, alors que la ligne en pointillés marque que la position argumentale dans la construction ne doit pas correspondre à un rôle thématique du verbe.

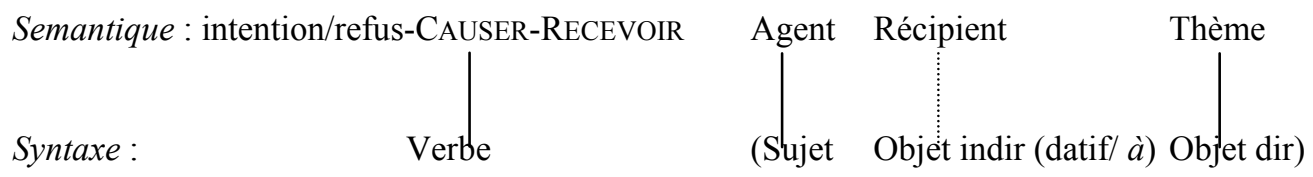

Figure 1

Il convient dès lors de s'interroger également sur la frontière entre argument et adjoint dans cette perspective constructionnelle.

Un dernier aspect à prendre en compte est la fonction pragmatico-discursive que remplit l'énoncé contenant la construction verbale. On observe ainsi à partir de l'exemple (3) que le complément locatif, tout en étant optionnel dans (d-e), ne saurait être supprimé ni déplacé vers la position initiale précédant le sujet préverbal si l'énoncé a pour fonction de poser l'existence du référent (a-b-c). Faut-il pour autant postuler une position argumentale de type locatif, dans la lignée de Kratzer (1995) ?

(3) a. Du sang sauta sur sa barbe blanche. (H. Pourrat)

b. *Du sang sauta.

c. *Sur sa barbe blanche, du sang sauta.

d. Le sang sauta sur sa barbe blanche.

e. Le sang sauta.

Dans la présente étude, nous nous interrogerons dans une triple perspective sur le statut du complément de la localisation spatiale, argument ou adjoint, en limitant notre examen aux verbes intransitifs. Dans la conception classique de la structure argumentale comme étant associée au verbe (Grimshaw 1992, Levin \& Rappaport Hovav 1995), quels sont les verbes pouvant être considérés comme ayant une structure argumentale dotée d'une position pour un complément locatif (\$2) ? Deuxièmement, existe-t-il des constructions verbales ayant une position argumentale locative (\$ 3) ? En troisième lieu, comment faut-il interpréter la contrainte de présence du complément locatif dans les énoncés à vocation existentielle (§ 4) ? Dans le prolongement de cette analyse, nous rendrons compte de la forme spécifique que revêt le prédicat d'existence il y $a$ en français (\$ 5). 


\section{Le complément de la localisation spatiale : argument ou adjoint des verbes de mouvement et de position?}

La localisation spatiale s'exprime souvent au moyen des compléments ayant le statut de circonstant. Auprès des verbes de position ou de mouvement, les compléments spatiaux présentent pourtant des contraintes plus fortes et semblent ainsi entrer dans la zone grise entre argument et adjoint.

Leur forme est rarement contrainte d'une manière absolue, même s'il existe des corrélations fortes entre certains verbes impliquant un mouvement directionnel ayant un point de départ ou d'aboutissement et le choix de la préposition introduisant le locatif. Ainsi un sondage effectué sur trois verbes directionnels, à savoir entrer, sortir, partir montre la variation des cooccurrences entre le verbe et un éventail de prépositions possibles $^{1}$. Il apparaît que entrer tend à sélectionner dans, que sortir se combine majoritairement avec de, alors que dans le cas de partir, on observe une concurrence entre les trois prépositions $\grave{a}$, de et pour pour marquer le mouvement directionnel.

\begin{tabular}{|c|c|c|c|}
\hline Préposition & entrer (263) & partir (195) & sortir (167) \\
\hline dans & 58 & 0 & 0 \\
\hline en & 21 & 0 & 0 \\
\hline$\grave{a}$ & 21 & 33 & 10 \\
\hline de & 0 & 30 & 66 \\
\hline pour & 0 & 15 & 0 \\
\hline en & 0 & 15 & 16 \\
\hline avec & 0 & 7 & 8 \\
\hline Total & 100 & 100 & 100 \\
\hline
\end{tabular}

Tableau 1 : Cooccurrences entre verbes et prépositions en \% (sur le total des cooccurrences $>10$ ) Analyse effectuée à l'aide de Syntex sur un fragment du quotidien Le Monde (2406252 mots)

En tout état de cause, cette corrélation est à concevoir en termes d'affinité sémantique plutôt que comme une contrainte stricte. Toute préposition introduisant un complément locatif peut en effet être remplacée par une préposition de sens voisin.

(4) a. Du Panthéon, je me rends à la place d'Italie par la rue Mouffetard. (E. \& J. Goncourt)

b. Les caravanes se rendaient vers les ports de la mer des Indes. (Michelet)

Quoique le complément locatif ne soit pas donc soumis à des contraintes formelles strictes, sa présence peut pourtant être requise. C'est le cas en particulier pour les verbes de position ou de direction dont le sens est relativement ténu, les cas les plus clairs étant être et aller.

(5) a. Un secret est dans l'air, va éclore (G. Bienne)

b. Un homme était près de moi et me touchait (C. Etcherelli)

c. Un homme est là, immobile dans le noir. (J. Joffo)

(6) a. Des flâneurs allaient devant eux, les mains dans leurs poches. (G. de Maupassant)

b. Des passants allaient sous la pluie. (F. Carco)

c. Elle roule. [...] Des fois, elle va à soixante! (V. Therame)

Si l'on admet qu'une localisation spatiale non instanciée mais de nature définie doit être considérée comme saturant une position argumentale, le nombre de verbes de position ou de mouvement ayant un argument locatif est beaucoup plus important. D'une manière globale, l'interprétation des énoncés contenant un verbe indiquant la position (rester) ou le mouvement directionnel sans préciser la manière du mouvement (entrer, sortir, partir, ...) suppose que le lieu ou la direction peuvent être identifiés. A cet effet sont activés les mécanismes de la deixis ou de l'anaphore. 
(8) Non loin de son dernier domicile, rue Caulaincourt, ma mère remarqua la vitrine d'un libraire de la rue $\underline{\text { Lamarck}}$, à la sortie du métro. Elle y vit un de mes livres. Sans hésiter, elle entra : « Je suis la mère de l'auteur. » (R. Grenier)

Les tests syntaxiques proposés pour déterminer le statut du complément locatif, argument du verbe ou adjoint, appuient le statut argumental du complément locatif auprès des verbes comme entrer, arriver, sortir, rester. Ils examinent la cohésion du lien entre le complément locatif et le verbe. Ainsi, d'après Reinhart (1983 : 68-72), alors qu'un adjoint peut être antéposé à un sujet interrogatif, un argument ne le permet pas, car il n'accepte pas d'être dissocié par rapport au groupe verbal. Comme l'observent Bresnan (1994: 82) et Kay (2005), ce test peut être mis à profit pour déterminer le statut du complément locatif, argument (9) ou adjoint (10).

(9) a. Pierre entre dans l'hôtel.

b. *Dans l'hôtel, qui entre ?

(10) a. Pierre nage dans la piscine de l'hôtel.

b. Dans la piscine de l'hôtel, qui nage?

Un second test, proposé par Lakoff \& Ross (1976) et également repris par Bresnan (1994), fait intervenir l'anaphore verbale faire de même : un adjoint peut être exclu de cette anaphore, un argument non. Selon ce critère, le complément locatif est argument du verbe entrer mais adjoint par rapport au verbe nager.

(11) a. Pierre est entré dans l'hôtel et Marie a fait de même *(dans le supermarché).

b. Pierre a nagé dans la piscine de l'hôtel et Marie a fait de même chez elle.

On observera au passage que le complément de localisation temporelle se comporte à l'égard de ce critère comme un adjoint dans les deux cas.

(12) a. Pierre est rentré à l'hôtel à cinq heures et Marie a fait de même à huit heures.

b. Pierre a nagé à cinq heures et Marie a fait de même à huit heures.

Les compléments de localisation spatiale à expression obligatoire auprès de verbes comme aller ou être ont dans la tradition grammaticale été analysés comme des arguments. Il est clair que la notion d'instanciation nulle définie ainsi que les tests mettant en évidence la cohésion du lien syntaxicosémantique du complément de localisation spatiale avec le verbe conduisent à donner une étendue plus importante au phénomène du complément locatif à statut d'argument. Parmi les verbes de position ou de mouvement, sont dotés d'une position argumentale locative tous les verbes exprimant la position ou le mouvement directionnel sans préciser la manière du mouvement. Les verbes correspondant à cette classe ont été analysés comme inaccusatifs: sur le plan de la syntaxe, le $\mathrm{SN}$ en position postverbale présente des propriétés objectales, dont le fait qu'il peut être mis en correspondance avec le pronom clitique régime en (Ruwet 1972, cf. Burzio 1986 pour l'italien), qu'il soit quantitatif (13a) ou génitif (13b) (Buchard 2009);

(13) a. Alors en arrivèrent / *nagèrent beaucoup d'autres.

b. Ses sculptures sont éphémères. Seules en sont restées des traces, des photographies et des souvenirs pour les spectateurs. (Buchard $2009: 311$ )

du point de vue du rôle thématique, ce SN postverbal correspond à un thème, c'est-à-dire à une entité dont se trouve prédiqué soit un état ou une localisation soit un changement d'état ou de localisation. Aussi peut-on proposer la représentation suivante :

(14) Verbe < thème loc > 


\section{Le complément de la localisation spatiale : argument ou adjoint des constructions à inversion locative et des constructions impersonnelles?}

La contrainte de présence du complément locatif a été retenue comme caractéristique de deux types de constructions, à savoir l'inversion locative (15) et la construction impersonnelle (16).

(15) A ses oreilles brillaient des anneaux argentés. (R. Sabatier)

?Des anneaux argentés brillaient.

(16) Il dort un chat au coin du feu.

*Il dort un chat.

Ces deux constructions ont été rapprochées : elles ont été caractérisées toutes deux en termes d'une discordance entre position, fonction syntaxique et rôle thématique, ce qui a donné lieu à des analyses du constituant nominal postverbal en termes de sujet inversé ou sujet réel ou logique (par opposition au sujet apparent ou fonctionnel, en position préverbale, revêtant la forme du pronom explétif ou du complément locatif) ou en termes de congruence entre position, fonction syntaxique et rôle thématique, d'autre part, ce qui a conduit à analyser le constituant nominal postverbal comme un objet, voire à analyser le complément locatif en position préverbale comme un sujet (cf. Bresnan (1994) pour le Chicheŵa). La discordance entre position, fonction syntaxique et rôle thématique serait liée à la prévalence de la structure informationnelle par rapport à la structure argumentale du verbe.

Dans le cadre de la présente étude, nous n'offrirons pas une analyse détaillée des constructions de l'inversion locative et de l'impersonnel. Nous nous limiterons à préciser la nature de la contrainte illustrée en (15) et (16) afin de déterminer si le complément locatif doit être considéré comme un argument pour chacune de ces deux constructions en français.

\subsection{L'inversion locative}

Sous le titre «Existentials and other locatives», Freeze (1992) avance l'hypothèse que l'inversion locative est la structure fondamentale permettant d'exprimer l'existence. Les propriétés dégagées pour cette structure en anglais par Bresnan (1994) sont compatibles avec cette analyse. Bresnan observe que l'inversion locative est limitée aux verbes inaccusatifs (17) ou constructions verbales inaccusatives, dont le passif (18) à condition que le complément d'agent ne soit pas exprimé (19). Sont exclus les verbes transitifs (19/20) ainsi que les verbes intransitifs de type inergatif (21).

(17) Among the guests was sitting my friend Rose.

(18) On the table has been placed a tarte Tatin.

(19) * On the table has been placed a tarte Tatin by Susan.

(20) * On the table has placed Susan a tarte Tatin.

(21) * On the table has placed a tarte Tatin Susan.

(22) *Among the guests was knitting my friend Rose.

Bresnan (1994: 80) synthétise ces contraintes sur la structure argumentale dans la généralisation suivante : l'inversion locative est possible quand le sujet peut être interprété comme l'argument dont la localisation, le changement de localisation ou la direction est prédiqué par le complément locatif. Le sujet est donc un thème au sens strict du terme. Elle propose la schématisation suivante :

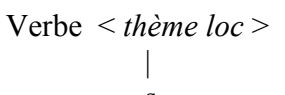

Si l'on admet que 'être dans l'espace', sur le mode dynamique ou statique, revient à 'exister', on peut comprendre la fonction existentielle de la construction à inversion locative, que lui attribue Freeze (1992). 
Peut-on admettre l'existence d'une construction équivalente en français susceptible de la même analyse ? Un exemple comme (24) semble conforter cette hypothèse : le constituant postverbal, tout en régissant à la manière d'un sujet l'accord en nombre du verbe ${ }^{2}$, présente des propriétés objectales : le pronom clitique en qui lui correspond est fondamentalement un pronom régime.

(24) a. A chaque mur pendaient plusieurs cadres.

b. A chaque mur en pendaient plusieurs.

La construction inversée du français ne présente toutefois pas les mêmes contraintes que celle de l'anglais. Mettons en évidence deux différences importantes.

La première concerne le constituant en position initiale : contrairement à ce que Bresnan a observé pour le complément en position initiale dans cette construction en anglais, le constituant initial de la construction française n'est pas fondamentalement associé à la localisation spatiale. Il peut aussi avoir trait au temps $(25 / 26 / 27)$ ou marquer un repère avec lequel s'effectue une mise en relation (28) (Erteshik-Shir 1997, Lahousse 2003).

(25) Soudain débouchent de toutes les portes des vaches, des génisses, des veaux, des taureaux.

(Chateaubriand)

(26) Déjà chante la reine. (R. Char)

(27) Alors parla le roi.

(28) A chaque cause correspond un traitement particulier. (Encyclopédie médicale)

De plus, son statut syntaxique n'est pas déterminé par la construction : peuvent figurer dans cette position aussi bien des arguments ou actants du verbe au sens strict du terme (régi et requis, par ex. (28)) que des adjoints ou circonstants (25/26/27) (Fuchs \& Fournier 2003, Fuchs 2006).

Enfin, le constituant initial peut être absent :

(29) Elle sonne. Arrive une infirmière. (F. Dolto, cité par Lahousse 2003 : 138)

Ainsi que le met en évidence Lahousse (2003), la construction exemplifiée par (29), dite 'd'inversion absolue' est contrainte : elle nécessite que puisse être restitué à partir du contexte et/ou des propriétés lexicales du verbe un cadre locatif ou un repère de mise en relation.

La deuxième différence concerne la nature du verbe : comme le montrent les exemples (26) et (27), le verbe entrant dans la construction dite d'inversion locative n'est pas nécessairement inaccusatif (Lahousse 2003, Buchard 2009) et, dans le cas d'un verbe inergatif, la correspondance du pronom clitique en avec le sujet est impossible.

(30) La chorale se mit en place. *Alors en chantèrent plusieurs filles.

Il n'est donc pas possible de soutenir l'analyse qu'en français la construction à inversion locative attribue au sujet inversé le statut de thème et que le complément locatif a pour rôle de prédiquer de ce sujet une localisation, un changement de localisation ou la direction. Par ailleurs, l'inversion locative en français n'est pas strictement existentielle.

Les données observées ont mis en évidence que la position initiale de la construction à inversion du sujet n'est pas réservée aux compléments de localisation spatiale. De plus, la relation syntaxico-sémantique qu'entretient le constituant initial avec le verbe est variable, car il peut être argument ou adjoint par rapport au verbe. Force est donc de conclure que la construction dite d'inversion locative en tant que telle n'est pas dotée d'une position argumentale pour un complément de localisation spatiale.

\subsection{La construction impersonnelle}

Tout comme la construction inversée, la construction impersonnelle a été associée à la prédication d'existence. Ce sémantisme a également été mis en rapport avec les contraintes imposées à la structure 
argumentale du verbe: seuls pourraient entrer dans la structure impersonnelle les verbes (31) ou constructions verbales de type inaccusatif, tel le passif (32) ou la construction pronominale de sens passif (33). Seraient donc exclus de cette structure les verbes transitifs et les verbes intransitifs de type inergatif.

(31) a. Un vent de nuit de forte haleine était venu.

b. Il était venu un vent de nuit de forte haleine. (J. Giono)

(32) a. Des milliers de soldats ont été tués durant la guerre.

b. Il a été tué des milliers de soldats durant la guerre.

(33) a. Beaucoup de nouvelles maisons se construisent dans ce quartier.

b. Il se construit beaucoup de nouvelles maisons dans ce quartier.

Il a toutefois été observé à répétition que la présence d'un complément de localisation spatiale permet de lever la contrainte de l'inaccusativité et autorise que s'intègrent dans la construction impersonnelle des verbes inergatifs (e.a. Jones 1996, Cummins 2000). Les occurrences de constructions impersonnelles comportant des verbes inergatifs se laissent répartir en deux classes :

- Le verbe exprime une activité ou procès caractéristique de l'entité correspondant au SN postverbal (Firbas 1966, Bolinger 1977).

(34) Il siffle des obus dans le ciel gris du nord. (Hériau $1980: 238$ )

(35) Il chante, au loin du bois, un carillon d'été. (Hériau $1980: 224$ )

- Le verbe exprime une activité ou procès qui se déroule habituellement dans le lieu évoqué (Jones 1996 : 126).

(36) Il nageait des enfants dans la piscine.

Par cette redondance sémantique par rapport au SN postverbal ou par rapport au complément locatif, le verbe subirait une désémantisation, lui permettant d'exprimer l'existence ou la venue à l'existence dans l'univers du discours (Guéron 1980), ce qui le rend compatible avec la construction impersonnelle. Ainsi l'exemple (36) pourrait être glosé par 'il y a des enfants dans la piscine'.

Quoique le verbe entrant dans la construction inaccusative ne soit pas nécessairement inaccusatif, il n'en reste pas moins que la construction impersonnelle est une structure syntaxique de nature inaccusative (Cummins 2000). A l'opposé du SN postverbal dans la construction inversée, le SN postverbal de la construction impersonnelle ne peut en effet plus être analysé comme un sujet, ce rôle étant dévolu au pronom $\mathrm{il}$. Le SN postverbal ne régit en effet pas l'accord du verbe (37b) et ne peut pas contrôler une proposition infinitive à statut d'adjoint (38b).

(37) a. Alors arrivèrent les tanks fascistes. (A. Malraux)

b. Il arriva des voitures de munitions. (Erckmann-Chatrian)

(38) a. Alors survinrent pour la huer ces hommes qui adorent un crucifié et possèdent toute certitude. (M. Barrès)

b. *Alors il survint pour la huer ces hommes qui adorent un crucifié.

Ce SN postverbal doit au contraire être analysé comme un objet. Il permet, en premier lieu, la pronominalisation par le pronom clitique objet en (39). Il est intéressant de noter avec Cummins (2000 : 234) que la pronominalisation par en est possible même avec les verbes non inaccusatifs (40), ce qui met en évidence la prévalence de la structure argumentale associée à la construction syntaxique par rapport à la structure argumentale associée au verbe en tant qu'unité lexicale.

(39) a. Il arrive plusieurs trains en même temps.

b. Il en arrive plusieurs en même temps. 
(40) a. Il vous arrivait, si vous dansiez, du cristal sur la tête et il en crissait sous vos semelles. (Hériau 1980 : 225)

Le statut de COD qu'acquiert le SN postverbal ressort d'une manière plus nette encore avec les quelques verbes transitifs qui peuvent entrer dans la construction impersonnelle à condition que leur objet soit exprimé sous forme de clitique. Si l'on admet que dans la construction impersonnelle, la position d'objet direct est occupée par le SN en position postverbale, on comprend pourquoi l'objet de prendre ne peut revêtir la forme du pronom clitique accusatif, mais doit s'exprimer sous la forme du pronom clitique datif lui (Cummins 2000 : 236).

(41) a. Une envie furieuse le prit de pousser les invités par les épaules.

b. Il lui/*le prit une envie furieuse de pousser les invités par les épaules. (d'après Atkinson $1973: 234$ )

Quel est le rôle exact du complément locatif dans les constructions impersonnelles ? Au complément locatif, utilisé en combinaison avec un verbe qui n'est pas intrinsèquement inaccusatif mais dont le sujet a des propriétés agentives, a été attribuée la faculté d'engendrer la désémantisation du verbe et de le transformer ainsi dans un prédicat d'existence de nature inaccusative, c'est-à-dire ayant comme argument unique un thème. A l'appui de cette hypothèse, Jones (1996) signale que le fait d'alourdir sémantiquement le prédicat verbal en insérant des adverbes caractérisant la manière dont le sujet agentif est impliqué dans le procès verbal diminue l'acceptabilité de la phrase ${ }^{3}$.

(36) a. Il nageait des enfants dans la piscine.

b. ?Il nageait sauvagement / bruyamment des enfants dans la piscine.

Il convient toutefois de noter en premier lieu que la désémantisation que peut engendrer un complément locatif n'est que partielle. Ainsi, le fait que le verbe flotter accepte comme sujet un nuage ou une vapeur en suspension dans l'air contribue à une appréhension métaphorique de un souvenir dans l'exemple (42d).

(42) a. Quelques nuages d'argent flottaient tout près des sommets pâles. (G. de Maupassant)

b. Il flotte des nuages, en ce regard, que je n'avais jamais aperçus. (J. Giraudoux)

c. Il y flotte une odeur d'amour. (G. de Maupassant)

d. Il y flottait un souvenir de Marceau, de ses amis que la tante trouvait «mauvais genre», des jolies petites amies. (R. Sabatier)

Par ailleurs, plusieurs faits demeurent inexpliqués dans le cadre de l'hypothèse attribuant au complément locatif la force de désémantiser le verbe et de lui permettre ainsi de fonctionner comme un verbe inaccusatif :

- Pourquoi le complément locatif est-il également indispensable quand la désémantisation du verbe ne se fonde pas sur la relation de redondance sémantique avec le complément locatif, mais s'opère par la relation de redondance sémantique que le verbe entretient avec le SN postverbal (exemples 34/35)?

- Pourquoi la présence du complément locatif est-elle même avec les verbes intrinsèquement inaccusatifs presque systématique?

- Pourquoi la construction impersonnelle est-elle parfois plus naturelle quand le complément locatif se présente sous la forme $y$ (Legendre \& Sorace $2003: 207$ )?

(43) a. L'autre jour, je suis allée à la rivière ; il $y$ pêchait des dizaines de personnes.

b. ?L'autre jour, il pêchait des dizaines de personnes dans la rivière.

Aussi convient-il de se demander si la présence exigée du complément locatif dans un exemple comme (36) est à mettre sur le compte de la construction impersonnelle ou si elle provient plutôt de la fonction de prédication existentielle de la construction impersonnelle. Afin de répondre à cette question, nous nous interrogerons sur la place et le rôle que tient le complément de localisation spatiale dans des énoncés où le prédicat a pour rôle de poser l'existence d'une entité sans néanmoins être intégré dans une construction impersonnelle. Nous examinerons à cet effet des énoncés dont le sujet est introduit par un article indéfini. 


\section{Le complément de la localisation spatiale dans les énoncés à sujet indéfini}

Relativement à la distinction entre énoncés catégoriques et énoncés thétiques telle qu'elle a été développée à la fin du XIX ${ }^{\mathrm{e}}$ siècle par les philosophes Brentano et Marty, puis élaborée dans une perspective linguistique par Kuroda (1972), les énoncés à sujet indéfini comme (44) et (45) sont thétiques.

(44) Un jeune homme inconnu était entré dans sa chambre. (P. Rambaud)

(45) Des marmitons tournaient des volailles à la broche sur un feu de brindilles. (P. Rambaud)

A la suite de Sasse (1987: 526), nous distinguerons deux types d'énoncés thétiques: les énoncés thétiques qui mettent au centre un événement dont ils posent l'existence (46) et les énoncés thétiques centrés autour d'une entité référentielle dont ils posent l'existence (47).

(46) Il pleut.

(47) Il arriva une lettre du propriétaire de l'appartement : il priait de vider les lieux. (E. Triolet)

Les énoncés à sujet indéfini peuvent relever des deux cas de figure : (44) constitue un énoncé thétique centré autour d'une entité, dit « existentiel », alors que (45) introduit un événement et, par ce biais, il pose également l'existence des référents des SN indéfinis impliqués dans cet événement. Dans le cadre de la présente étude, limitée aux verbes intransitifs, nous étudierons exclusivement les énoncés thétiques du premier type, centrés autour d'une entité référentielle dont l'existence est posée.

A partir d'une liste de 41 verbes intransitifs, nous avons extrait de Frantext un corpus de 1115 exemples dont le sujet est introduit par un article indéfini, afin de dégager les stratégies permettant d'introduire une entité référentielle. Le tableau 2 montre la fréquence de ces verbes dans la construction à sujet indéfini.

\begin{tabular}{|c|c||c|c||c|c|}
\hline Verbes & $\begin{array}{c}\text { Fréquence } \\
\text { d'occurrence } \\
\text { en } \%\end{array}$ & $\begin{array}{c}\text { Verbes } \\
\text { (suite) }\end{array}$ & $\begin{array}{c}\text { Fréquence } \\
\text { d'occurrence } \\
\text { en } \%\end{array}$ & $\begin{array}{c}\text { Verbes } \\
\text { (suite) }\end{array}$ & $\begin{array}{c}\text { Fréquence } \\
\text { d'occurrence } \\
\text { en \% }\end{array}$ \\
\hline Venir & 16,0 & Voler & 2,2 & Trotter & 0,4 \\
\hline Arriver & 8,7 & Siffler & 1,7 & Trottiner & 0,3 \\
\hline Entrer & 8,5 & Marcher & 1,6 & Serpenter & 0,2 \\
\hline Tomber & 8,4 & Circuler & 1,5 & Boitiller & 0,1 \\
\hline Sortir & 7,3 & Errer & 1,1 & Détaler & 0,1 \\
\hline Courir & 7,2 & Sauter & 1,1 & Echapper & 0,1 \\
\hline Eclater & 5,9 & Habiter & 0,9 & Etinceler & 0,1 \\
\hline Couler & 4,2 & Survenir & 0,8 & Flâner & 0,1 \\
\hline Rester & 3,3 & Approcher & 0,6 & Fleurir & 0,1 \\
\hline Partir & 3,2 & Ramper & 0,5 & Nager & 0,1 \\
\hline Rouler & 3,1 & Craquer & 0,4 & Scintiller & 0,1 \\
\hline Flotter & 3,0 & Menacer & 0,4 & Se précipiter & 0,1 \\
\hline Briller & 2,8 & Dévaler & 0,4 & Zigzaguer & 0,1 \\
\hline Glisser & 2,5 & Galoper & 0,4 & & \\
\hline
\end{tabular}

Tableau 2 : Fréquence relative des verbes intransitifs dans les énoncés à sujet indéfini (en un, du, des)

L'examen de ces verbes en corpus révèle la prédominance nette de la localisation dans l'espace comme stratégie d'ancrage référentiel. En effet, comme le montre déjà le tableau 2, du point de vue du lexique verbal, les verbes les plus fréquents sont ceux qui encodent d'une manière intrinsèque un mouvement 
directionnel vers un lieu ou à partir d'un lieu, les verbes venir, arriver, entrer, tomber, sortir totalisant à eux seuls la moitié des occurrences.

Par ailleurs, environ 50\% des exemples (562 exemples sur le total des 1115 exemples) comporte un complément de localisation spatiale ou directionnel explicite. L'étude de la combinatoire des verbes et des compléments locatifs, conduit à donner plus de poids encore à cette présence du complément de la localisation spatiale : le complément locatif est en effet moins fréquent auprès des verbes comme arriver, entrer, qui comportent une position argumentale locative pouvant être saturée par une instanciation nulle définie (cf. § 2) (48) mais il est attesté plus systématiquement auprès des verbes qui n'ont pas une telle position argumentale (49), dont les verbes de mouvement non directionnels indiquant la manière (50).

(48) Un jeune homme entra. (V. Hugo)

(49) Une femme travaille devant la maison. (J. Chardonne)

(50) Des gouttes flasques glissaient $d u$ toit. (R. Martin du Gard)

Le graphe 1 ci-dessous fait ressortir la fréquence plus élevée du complément de localisation spatiale auprès des verbes précisant la manière du mouvement par rapport aux verbes indiquant la direction du mouvement.

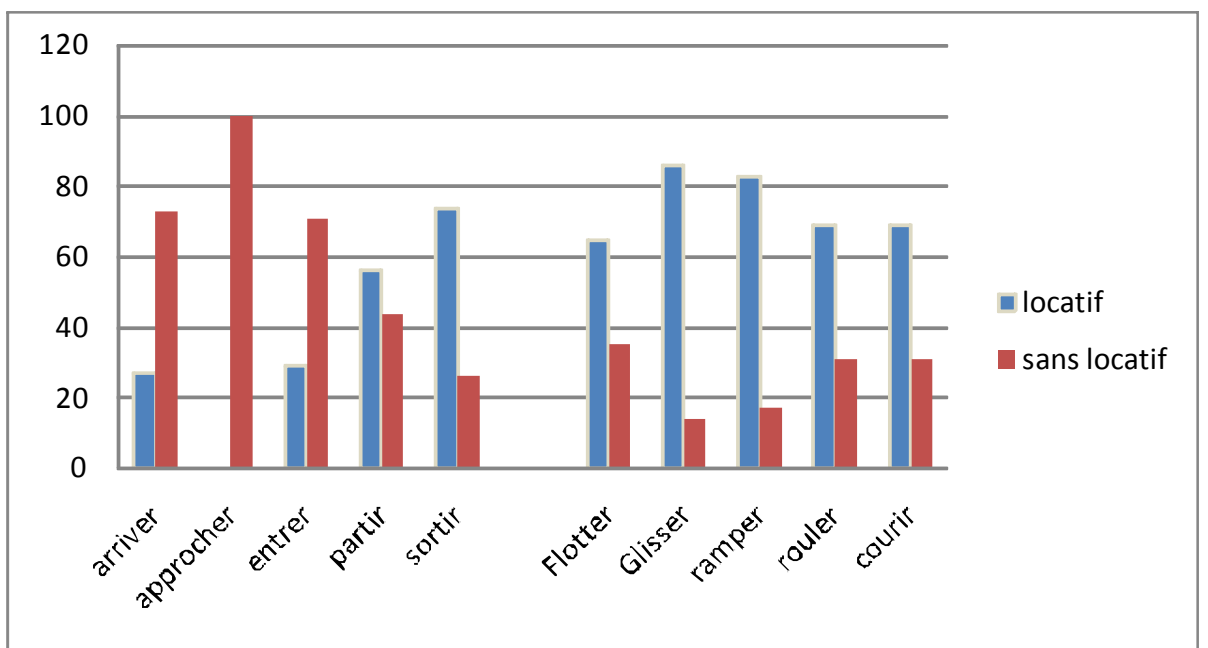

Graphe 1: Cooccurrence $\mathrm{du}$ verbe de mouvement et $\mathrm{du}$ complément locatif dans les énoncés à sujet indéfini, en \%

Dans les énoncés à sujet indéfini comportant un verbe marquant la manière du mouvement sans indiquer la direction, les compléments de localisation spatiale sont non seulement très fréquents, ils peuvent en plus entretenir un rapport de cohésion fort avec le verbe, manifesté par des contraintes positionnelles. En particulier quand le complément spatial indique le point d'aboutissement ou le point d'origine du mouvement et conduit ainsi à concevoir le mouvement comme étant directionnel, il est, à l'instar d'un argument, ni supprimable, ni déplaçable vers la position initiale précédant le sujet préverbal et le verbe.

(51) a. Du sang sauta sur sa barbe blanche. (H. Pourrat)

b. *Du sang sauta.

c. ??Du sang sauta vigoureusement.

d. * Sur sa barbe blanche, du sang sauta. (cf. Sur sa barbe blanche sauta du sang)

L'étude de corpus met en évidence, face à cette présence massive du complément de localisation spatiale, l'extrême rareté du complément de la localisation temporelle dans les énoncés posant l'existence d'une entité. En effet, celui-ci est attesté seulement dans $1 \%$ des exemples. Les exemples (52) et (53) mettent en évidence que l'ancrage temporel ne permet pas à lui seul de conférer à un verbe de position ou de mouvement non directionnel un sens existentiel. 
(52) a. Des jouets et des légos traînaient dans la pièce.

b. ??Des jouets et des légos traînaient ce matin.

(53) a. Un homme s'avança vers eux.

b. ??Un homme marcha pendant l'après-midi.

Afin de comprendre cette dissymétrie entre localisation spatiale et localisation temporelle en tant que stratégie d'ancrage référentiel, il convient de s'attarder sur la manière dont les entités référentielles sont conceptualisées dans la langue. Dans une perspective philosophique, Zemach (1979) soutient qu'il y a quatre manières différentes de concevoir des entités ayant une extension spatio-temporelle ou quatre ontologies possibles, que nous définirons par rapport à la figure suivante.

Figure 2

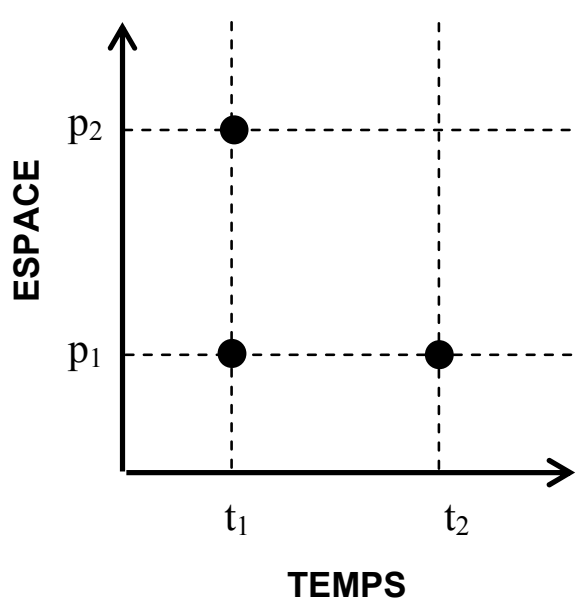

- La première ontologie est celle des événements, qui sont conceptualisés comme étant discontinus tant dans la dimension temporelle que dans la dimension spatiale. En effet, si une explosion se produit au même moment $t_{l}$ à deux lieux distincts et disjoints $p_{1}$ et à $p_{2}$, on considérera qu'il y a eu deux explosions. De la même manière, si une explosion se produit au même endroit $p_{1}$ à deux moments distincts et disjoints $t_{l}$ et $t_{2}$, il sera question de deux explosions. Cette ontologie, utilisée naturellement pour parler des événements, peut également être transposée aux domaines des choses. Ainsi, la conception des individus comme pouvant être décomposés en «stages » de cet individu est cruciale dans la théorie de Carlson (1978). Il est admis en effet que dans un exemple comme Un chien est en train d'aboyer le prédicat ne se rapporte pas à l'individu en tant que tel, mais à une tranche temporelle de cet individu.

- La deuxième ontologie est celle des choses, qui sont conceptualisées comme étant continues dans la dimension temporelle, mais discontinues dans la dimension spatiale. Les entités dénotées par des noms comme chaise ou chien sont d'habitude considérées comme continues dans la dimension temporelle car si nous observons un chien ou une chaise en tel lieu précis $p_{1}$ à deux moments distincts $t_{1}$ et $t_{2}$, rien ne nous empêche de considérer qu'il s'agit de la même chaise ou du même chien. Une différence de localisation temporelle n'est donc pas suffisante pour établir qu'il s'agit de deux entités distinctes. Les entités dénotées par des noms comptables concrets comme chaise ou chien sont par ailleurs conçues comme discontinues ou bornées dans la dimension spatiale : si j’observe à tel moment précis $t_{1}$ un chien à tel endroit $p_{1}$ et à tel autre endroit $p_{2}$, il est impossible de considérer qu'il s'agit du même chien. Il s'agit nécessairement de chiens différents.

- La troisième ontologie, dont les entités sont considérées comme étant continues dans la dimension spatiale et discontinues dans la dimension temporelle, peut être illustrée par des noms comme la crise bancaire, la Révolution industrielle. Si la crise bancaire se produit à tel moment $t_{1}$ en deux lieux distincts et disjoints $p_{1}$ et $p_{2}$, nous dirons qu'il s'agit de la même crise bancaire. En revanche, si la crise bancaire se manifeste à tel endroit $p_{I}$ à deux moments distincts et disjoints $t_{l}$ et $t_{2}$, nous dirons qu'il s'agit de deux crises bancaires différentes. Les termes qui font référence à ce type d'entité sont de nature temporelle ('ère', 'période'), quoiqu'il n'existe pas vraiment, à notre connaissance, de terme générique pour le catégoriser. 
- La quatrième ontologie est celle des types, conçus comme continus tant dans la dimension temporelle que dans la dimension spatiale. Il est possible d'évoquer des types par des SN génériques comme le contribuable, l'ours polaire. Par rapport à ces types, les individus situés dans le temps et dans l'espace peuvent tout au plus être considérés comme des instances exemplifiant le type.

Cette présentation rapide des quatre ontologies doit suffire pour comprendre la dissymétrie entre temps et espace dans les procédures d'ancrage référentiel, illustrée par les exemples (52) et (53).

(52) a. Des jouets et des légos traînaient dans la pièce.

b. ??Des jouets et des légos traînaient ce matin.

(53) a. Un homme s'avança vers eux.

b. ??Un homme marcha pendant l'après-midi.

Si l'on admet que la langue conceptualise les entités référentielles conformément à l'ontologie des choses, comme continues dans le temps, mais discontinues ou circonscrites dans l'espace, on comprend pourquoi l'ancrage dans l'espace a une fonction individuante qui ne saurait être garantie par un ancrage dans le temps. En effet, comme une chose est dotée d'une continuité dans le temps, une localisation temporelle précise ne permet pas d'établir son identité. Mais étant donné qu'elle est circonscrite ou liée dans l'espace, le fait de prédiquer à son propos qu'elle est dans l'espace revient à dire qu'elle existe en tant qu'entité individuelle. C'est ainsi que la prédication d'existence prend le plus souvent la forme d'un verbe de localisation ou de mouvement accompagné d'un complément de localisation spatiale, à moins que cette localisation ne puisse être restituée à partir du contexte ou de la situation d'énonciation.

Les données du corpus mettent en évidence que les prédicats posant l'existence d'une entité référentielle correspondant à un nom de matière sont soumis à la même contrainte de la localisation spatiale (cf. également Van de Velde 1996 et Theissen 1997).

(54) a. Du sang suintait de sa blessure.

b. ??Du sang coula au bout de cinq minutes.

Il est plus étonnant encore d'observer que même dans le cas des entités référentielles qui se présentent comme circonscrites dans la dimension temporelle tels orage, catastrophe, silence, le prédicat d'existence comporte le plus souvent un complément de localisation spatiale et que l'expression du complément temporel (55 à 61), tout en étant attesté, est plus rare (62-63).

(55) Une petite pluie fine tremblotait dans l'air. (Erckmann-Chatrian)

(56) Un orage grondait au loin. (G. Duhamel)

(57) Des explosions minuscules retentirent de tous côtés. (J.-M. Le Clézio)

(58) Des accidents énormes ont jailli $d u$ sein de cette vallée. (G. Flaubert)

(59) Un silence lourd plana sur la pièce enfumée. (G. Pérec)

(60) Un grand silence tomba sur la salle. (J. d'Ormesson)

(61) Un sourire mince flottait sur ses lèvres jointes. (A. Robbe-Grillet)

(62) Une tempête furieuse éclata durant le souper. (G. Sand)

(63) Un hennissement éclata soudain. (Th. Gauthier)

C'est comme si la langue alignait ces entités référentielles également sur l'ontologie des choses.

Quoique la localisation dans l'espace soit la stratégie dominante d'ancrage référentiel, il convient de signaler deux autres stratégies de prédication d'existence également attestées dans le corpus :

- Un prédicat verbal qui exprime un comportement stéréotypique de l'entité référentielle correspondant au sujet indéfini, qui soit conforme aux attentes et n'apporte ainsi pas d'information nouvelle, peut servir à poser simplement l'existence de cette entité référentielle. Dans la même perspective, on trouve souvent un empilage de prédicats exprimant une scène stéréotypique.

(64) a. Un chien aboya. (R. Fallet)

b. Un chien aboyait et gémissait, et plus prompt qu'eux, il vint à diverses reprises gratter à la porte. (G. Sand) 
(65) Des chefs venaient, discouraient, essayaient de persuader ces hommes. (M. Van der Meersch)

(66) Une foule flânait, fluctuait, traînait ou s'affairait. (M. Van der Meersch)

(67) Un obus tomba, tonna, flamba, éclata... écrabouilla le petit cuistot. (R. Benjamin)

- Un autre cas de figure est représenté par le cas où l'entité référentielle sujet du verbe de mouvement devient le support d'une prédication seconde. Le degré d'intégration de cette prédication seconde par rapport au prédicat verbal central peut varier. Si le verbe de mouvement est inaccusatif, le prédicat second peut être un attribut (Buchard 2006).

(68) Des chameaux tombèrent morts d'épuisement.

(69) Un domestique entra tout effaré, racontant que le feu venait de prendre chez la fille de Madame.

(70) Un homme vint qui avait à la fois les sens d'un artiste et le cour d'un prophète et qui fit jaillir son poème du choc de la passion et du savoir. (E. Faure)

Cette prédication seconde prend toutefois le plus souvent un caractère appositif.

(71) Des balles glissent, flâneuses, vers le Nord. (M. Genevoix)

(72) Un monsieur entra, de taille moyenne, plutôt mince, à peine grisonnant, - sauf une curieuse mèche, presque blanche - avec un air de réflexion pénétrante, extrêmement calme.

(73) Des promeneuses erraient encore, épaves rejetées par la nuit. (Montherlant)

La présente étude a été limitée aux verbes intransitifs. Un examen des verbes transitifs mettrait toutefois de la même manière en évidence l'importance de la localisation spatiale dans la prédication d'existence. Dans les phrases posant l'existence du sujet indéfini, la fréquence des prédicats transitifs où la localisation spatiale se fait par le biais de l'objet direct est en effet frappante.

(74) Une averse fouettait les vitres à croisillons. (B. Poirot-Delpech)

(75) Une course précipitée troubla la rue déserte. (V. Hugo)

(76) Un costume gris sortait la cour. (E. Orsenna)

\section{La grammaticalisation de l'expression de la localisation spatiale dans les prédicats d'existence II $\boldsymbol{y}$ a et le choix du verbe avoir}

L'importance cruciale de la localisation spatiale dans la prédication d'existence permet de comprendre pourquoi dans beaucoup de langues le prédicat existentiel de base contient un adverbe de localisation spatiale, comme l'adverbe $y$ en français, l'adverbe $c i$ en italien, l'adverbe there en anglais, l'adverbe er en néerlandais (Lyons 1967). Par ailleurs, en allemand exister se traduit comme dasein ('être là').

L'ancien français nous permet d'observer le prédicat d'existence en cours de grammaticalisation. On y observe une concurrence entre plusieurs formes d'expression. Tant le verbe être (77) que le verbe avoir (78) sont utilisés. Quand la tournure impersonnelle fait intervenir le verbe avoir, le constituant nominal postverbal se trouve le plus souvent au cas régime et se présente ainsi comme un objet (79) (Buridant $2000: \S 53$ ).

(77) Forz Renouart ainz ne $f u$ si fort home (Aliscans, 370) [En dehors de Rainouart, jamais il n'y eut d'homme si fort]

(78) En la forest du Marés avoit une montaigne grans et merveilleuse, u il avoit dessus fermé un castel mout anchien. (Tristan en prose, I, $177: 13-15$ )

[Dans la forêt du Morois, il y avait une haute montagne, extraordinaire, sur laquelle il y avait un château-fort très ancien]

(79) N'i out un nen parolt (Voyage de Charlemagne, 812)

[il n'y eut pas un qui n'en fasse des commentaires]

L'ancien français possède par ailleurs une structure avec le verbe avoir pour affirmer l'existence d'une propriété dont le site de localisation est humain. 
A ce stade de l'évolution, l'adverbe locatif $i$ n'est pas encore obligatoire. Au contraire, comme le fait remarquer Buridant (2000: §317), «en règle générale, avec avoir l'adverbe $i$ fait défaut au sens d'existence si la phrase contient un autre complément de lieu, comme $c i$ dans $c i$ a, mais les exceptions ne sont pas rares. L'on peut trouver ainsi ci i $a »$.

La concurrence entre être et avoir en tant que prédicat d'existence se maintient en moyen français.

(81) Il fut jadis et a esté un phillosophe, qui Secons Avoit nom (Eustache Deschamps, Le Miroir de Mariage [1385])

[Il y eut jadis un philosophe qui portait le nom de Secons]

(82) Oultre de la forest ost une ostelerie (Tristan de Nanteuil, Sinclair [circa 1350])

[Au-delà de la forêt, il y avait une auberge]

Alors que l'italien retient la structure « ADV LOC + être + sujet inversé » (c'è / ci sono + SN), le français va finir par opter pour la structure « pronom explétif sujet + ADV LOC + avoir + objet » (il y a + SN). Par ailleurs, l'expression de l'adverbe locatif $y$ devient obligatoire.

Afin d'expliquer que le français a fini par opter pour le verbe avoir dans la formation du prédicat d'existence, on peut invoquer le figement progressif de l'ordre des constituants. Dès le XIII ${ }^{\mathrm{e}}$ siècle, l'ordre « verbe - objet nominal (VO)» se généralise (Marchello-Nizia 2006 : 47). A partir de la fin du $\mathrm{XIV}^{\mathrm{e}}$ siècle, l'expression du sujet sous forme de pronom devient obligatoire en l'absence de sujet lexical et le sujet tend à se trouver en position préverbale (Combettes 1985, Prévost 1997). La structure « pronom explétif sujet + ADV LOC + avoir + objet » permet de positionner le SN indéfini, conformément à son statut informationnel de rhème, en position postverbale, tout en respectant le nouvel ordre des mots SVO. L'italien, en revanche, a maintenu une plus grande souplesse dans l'ordre des constituants et permet encore aujourd'hui l'inversion du sujet avec une fonction rhématisante. La structure « ADV LOC + être + sujet inversé » lui convient ainsi en tant que prédicat d'existence.

Cette hypothèse demande à être vérifiée par un examen empirique détaillé. Elle peut néanmoins s'appuyer sur le fait que le recul du sujet en position inversée au cours du moyen français a déclenché d'autres évolutions syntaxiques, telles la réanalyse du sujet inversé en attribut dans la structure copulative (ce suisje > c'est moi cf. Foulet 1920, Carlier 2004-2005)

\section{Conclusion}

Quoique la distinction entre argument et adjoint ait une longue tradition grammaticale et s'appuie sur des critères généralement acceptés, la présente étude a mis en évidence la difficulté de déterminer le statut précis du complément de la localisation spatiale par rapport à cette distinction. Le complément de la localisation spatiale, tout en étant caractérisé par une certaine liberté du point de vue de sa forme, peut être soumis à des contraintes de présence et de position. Afin de comprendre ces contraintes, il convient de prendre en compte trois niveaux.

- Le premier niveau est celui de la structure argumentale du verbe. En combinaison avec les verbes marquant la position ou le déplacement de leur sujet, sans préciser la manière du mouvement, le complément locatif, sans être obligatoirement exprimé, présente néanmoins un lien de cohésion fort avec le verbe, ce dont témoigne la contrainte positionnelle. Si l'on admet qu'un élément non instancié mais nécessairement défini est un argument, on est amené à conclure que tous les verbes de ce groupe ont une position argumentale locative.

- Le deuxième niveau est celui de la construction verbale. L'étude de la construction impersonnelle a mis en évidence la prédominance de la structure argumentale associée à la construction par rapport à celle associée au verbe. La fonction existentielle de l'impersonnel permet de comprendre la fréquence du complément locatif, y compris pour des verbes dépourvus d'argument locatif. 
- C'est ainsi que nous nous sommes intéressées enfin aux contraintes provenant de la fonction pragmaticodiscursive de l'énoncé : les énoncés qui ont pour fonction de poser l'existence d'une entité référentielle expriment très souvent cette opération en affirmant que cette entité est dans l'espace. Aussi le complément locatif peut-il revêtir un caractère obligatoire et être contraint positionnellement dans les phrases à sujet indéfini.

Le rôle fondamental de l'espace dans la prédication d'existence permet de comprendre pourquoi l'adverbe de localisation spatiale est un élément constitutif du prédicat d'existence dans la plupart des langues. Nous avons retracé le processus de figement du prédicat d'existence il y a «pronom explétif sujet + ADV LOC + avoir + objet» du français et, en le comparant avec le prédicat d'existence de l'italien c'èl ci sono «ADV LOC + être + sujet », nous avons rendu compte de sa forme spécifique.

Cette étude n'offre qu'une amorce de l'analyse du statut du complément de la localisation spatiale, entre argument et adjoint. Elle met néanmoins en place les principes pour la recherche ultérieure sur la question: l'analyse du statut du complément de la localisation spatiale doit prendre en compte trois niveaux, le verbe en tant qu'unité du lexique, son insertion dans une construction verbale et sa fonction pragmatico-discursive. Ce n'est qu'en distinguant ces trois dimensions et en dégageant leur articulation que l'on peut avancer dans la compréhension du rôle fondamental que joue la localisation spatiale dans la langue.

\section{Références bibliographiques}

Atkinson J.C. (1973). The two forms of subject inversion in French. The Hague: Mouton.

Bolinger D. (1977). Form and Meaning, London: Longman.

Bonami O \& D. Godard. (2001). Inversion du sujet, constituance et ordre des mots. In: J. M. Marandin Ed., Cahier Jean-Claude Milner, 117-174. Paris : Verdier.

Bourigault, D. (2007). Un analyseur syntaxique opérationnel : SYNTEX. Thèse d'habilitation à diriger des recherches, Université Toulouse 2-Le Mirail

Bresnan J. (1994). Locative Inversion and the Architecture of Universal Grammar. Language, 70, 72-131.

Buchard A. (2006). Pour une analyse unitaire de l'attribut du sujet et de l'attribut de l'objet. Travaux de linguistique, $53,67-89$.

Buchard A. (2009). Etre + participe passé en tant que marqueur d'aspect et de structure argumentale. Thèse de Doctorat, Université de Valenciennes.

Buridant C. (2000). Grammaire nouvelle de l'ancien français. Paris : SEDES.

Burzio L. (1996). Italian Syntax: A Government-Binding Approach. Dordrecht: Reidel.

Carlier A. (2004-2005). 'Ce sont des Anglais' : un accord avec l'attribut? l'Information grammaticale 103, 13-18; 104, 4-14.

Carlier A. (2005). L'argument davidsonien : un critère de distinction entre les prédicats 'stage level' et les prédicats 'individual level' ? Travaux de linguistique 50, 13-35.

Carlson G.N. (1978). Reference to Kinds in English, Bloomington: Indiana University Club.

Combettes B. (1988). Recherches sur l'ordre des éléments dans la phrase en moyen français. Thèse de pour le Doctorat d'Etat, Université de Nancy.

Croft W. (2001). Radical construction grammar. Oxford : UP.

Cummins S. (2000). The unaccusative hypothesis and the impersonal construction in French. Canadian Journal of Linguistics, 45, 225-251.

Dowty D. (2003). The dual analysis of adjuncts/complements in Categorial Grammar. In: Lang E., Maienborn C., Fabricius-Hansen Eds. Modifying Adjuncts, 33-66. Berlin, New York: Mouton-De Gruyter.

Erteschik-Shir, N. (1997), The Dynamics of Focus Structure, Cambridge: UP. 
Fillmore Ch. \& Kay P. (1996). Course Book of Constructional Grammar. Manuscript, University of California at Berkeley Department of linguistics.

Firbas J. (1966). Non-thematic subjects in contemporary English. Travaux de linguistique de Prague, 2, 239-256.

Foulet L. (1920). Comment on est passé de ce suis je à c'est moi. Romania, XLVI, 46-83.

Freeze R. (1992). Existentials and other locatives. Language, 68, 553-595.

Fuchs C. (2006). "Locatif spatial initial et position du sujet nominal: Pour une approche topologique de la construction de l'énoncé », Lingvisticae Investiagationes, 29, 1, 61-74.

Fuchs C. \& Fournier N. (2004). Du rôle cadratif des adverbiaux initiaux selon la position du sujet nominal, Travaux de linguistique, 47, 79-109.

Goldberg A. E. (1995). A construction grammar approach to argument structure. Chicago : UP.

Goldberg A. E. (2006). Constructions at work. Oxford : UP.

Grimshaw J. (1990). Argument Structure. Cambridge, Mass.: MIT Press.

Guéron J. (1980). On the syntax and semantics of the PP-extraposition. Linguistic Inquiry, 11, 637-678.

Hériau M. (1980). Le verbe impersonnel en français moderne. 2 vols. Lille : Atelier de reproduction des thèses.

Herschensohn J. (1982). The French presentationals as base generated structure. Studies in Languag, 6, 193-219.

Hoekstra \& Mulder R. (1990). Unergatives as copular verbs: locational and existential predications. The Linguistic Review, 7, 1-79.

Jones W. (1996). Foundations of French Syntax. Oxford: UP.

Kay P. (2005). Argument Structure Constructions and the Argument-Adjunct Distinction. In M. Fried and H. Boas Eds. Grammatical Constructions: Back to the Roots, Amsterdam : Benjamins, 71-98.

Kratzer A. (1995). Stage-level and individual-level predicates. In: Carlson G. N. et al. Eds, The Generic Book, Chicago : UP, 125-175.

Kuroda S.-Y. (1973). Le jugement catégorique et le jugement thétique: exemples tirés de la syntaxe japonaise. Langages, 30, 81-110.

Lahousse K. (2003). La distribution de l'inversion nominale en français dans les principales non interrogatives et les subordonnées circonstancielles. Lingvisticae Investigationes, 26, 1, 123-158.

Lakoff G. \& Ross J. R. (1976). Is deep structure necessary? In J. D. McCawley Ed. Syntax and semantics, 7, 159-164.

Lazard G. (1996). L'actance. Paris: PUF.

Legendre G. \& Sorace A. (2003). Auxiliaires et intransitivité en français et dans les langues romanes. In : Godard D. Ed. Les langues romanes : problèmes de la phrase simple. Paris : Ed. CNRS.

Levin B. \& Rappaport Hova M. (1996). Unaccusativity: At the syntax-semantics interface. Cambridge Ms: MIT Press.

Marandin J.-M. (2001). Unaccusative inversion in French. In D’hulst Y., Rooryck J. \& Schroten J. Eds. Romance Languages and Linguistic Theory 1999: Selected papers from Going Romance 1999, Amsterdam : Benjamins, 195-222.

Marchello-Nizia Ch. (2006). Le français en diachronie : douze siècles d'évolution. Paris : Ophrys.

Prévost S. (2001). La postposition du sujet en français aux $15^{e}$ et $16^{e}$ siècles. Paris : CNRS Editions.

Reinhart T. (1983). Coreference and bound anaphora: A restatement of the anaphora questions. Linguistics and Philosophy, 6. 47-88.

Ruwet N. (1972). Théorie syntaxique et syntaxe du français. Paris: Seuil.

Sarda L. (2000). L'expression du déplacement dans la construction transitive directe. Syntaxe et sémantique, 2, 212237.

Sasse H.J. (1987). The thetic-categorical distinction revisited, Linguistics, 25, 511-580. 


\section{Syntaxe}

DOI $10.1051 / \mathrm{cmlf} / 2010251$

Theissen A. (1997). Quand le partitif peut-il être sujet ? Verbum, XIX(3), 339-354.

Van de Velde D. (1996). Le Spectre nominal, Louvain : Peeters.

Zemach E. (1979). Four ontologies. In Pelletier F.J. Ed. Mass Terms : some philosophical problems, Dordrecht : Reidel, 63-87.

Sources des exemples : Frantext, DMF, Buridant (2000) pour les exemples (77) à (79)

${ }^{1}$ Ce sondage a été réalisé sur un corpus journalistique (Le Monde, décembre 2000, 2406252 mots) à l'aide de Syntex, un analyseur syntaxique repérant les relations recteur-régi (Bourigault 2007).

${ }^{2}$ Comme le notent Bonami et al. (2001) pour l'inversion en contexte d'extraction, l'accord avec le SN en position postverbal concerne le nombre mais non le genre (Bonami et al. 2001).

A cet interrogatoire *assistiez/assistèrent Pierre et toi.

${ }^{3}$ Les jugements d'acceptabilité sont variables d'après les locuteurs: alors que certains locuteurs refusent la construction impersonnelle avec un verbe inergatif même dans le cas de (36a), d'autres locuteurs ont tendance à accepter (36b). Dans tous les cas, (36b) est néanmoins ressenti comme moins naturel que (36a). 